FedUni ResearchOnline

https://researchonline.federation.edu.au

This is the peer-reviewed version of the following article:

Wang, Y. (2014). Amubulatory blood pressure may be designed as the primary efficacy outcome in clinical trials on renal denervation. International Journal of Cardiology 176(3): 1262-1263

Which has been published in final form at:

https://doi.org/10.1016/j.ijcard.2014.07.192

Copyright $@ 2014$ Elsevier Inc. All rights reserved. 


\section{Ambulatory blood pressure may be designed as the primary efficacy outcome in clinical trials on renal denervation}

Yutang Wang, $\mathrm{PhD}$.

The Vascular Biology Unit, Queensland Research Centre for Peripheral Vascular Disease, School of Medicine and Dentistry, James Cook University, Townsville, Queensland 4811, Australia

Corresponding author: Yutang Wang. The Vascular Biology Unit, Queensland Research Centre for Peripheral Vascular Disease, School of Medicine and Dentistry, James Cook University, Townsville, Queensland 4811, Australia. Tel: +61 74781 3155; fax: +61 74781 3652; E-mail address: yutangwang000@gmail.com

Short title: Ambulatory blood pressure and renal denervation 
To the Editor

Renal denervation has been approved as a standard treatment option for resistant hypertension in many countries since 2010. The efficacy of renal denervation in lowering blood pressure is controversial [1], emphasizing that more research needs to be conducted to investigate the effect of renal denervation on blood pressure. In previous clinical trials on renal denervation, blood pressure was mainly monitored by office blood pressure; when ambulatory blood pressure data were available, such data were often incomplete because ambulatory blood pressure was not designed as the primary efficacy outcome.

A large amount of evidence suggests that ambulatory blood pressure may be a better inclusion criterion, and changes in ambulatory blood pressure may be designed as the primary efficacy outcome in clinical trials on renal denervation for the following reasons:

\section{Limitations in office blood pressure measurement}

Office blood pressure measurement is well known to produce a white coat effect. Therefore, measurement of office blood pressure, though standard and well-established, is often misleading. For example, 37.5\% of patients with resistant hypertension based on office blood pressure were found to have white coat hypertension [2] which may not contribute to cardiovascular events [3]. It has been shown that renal denervation does not lower ambulatory blood pressure in subjects with white coat hypertension [4]. Therefore, performing renal denervation in subjects with white coat hypertension may raise ethical concerns because long-term side effects of renal denervation are unknown [5].

In addition, office blood pressure measured at a certain time point of the day may not accurately reflect the blood pressure levels in patients with resistant hypertension who take $\geq 3$ antihypertensive drugs. Office blood pressure can change dramatically after the intake of antihypertensive drugs. For example, the office systolic blood pressure in a renal denervated 
patient decreased from $177 \mathrm{~mm} \mathrm{Hg}$ at baseline to 97, 94 and $140 \mathrm{~mm} \mathrm{Hg}$ at 1, 2 and $6 \mathrm{~h}$ after the intake of antihypertensive drugs, respectively [6]. Therefore, without information on when patients take antihypertensive drugs and when blood pressure is measured, office blood pressure would have a limited power accurately to assess the effect of renal denervation on blood pressure.

\section{The superiority of ambulatory blood pressure monitoring}

Ambulatory blood pressure monitoring is regarded as the gold standard to diagnose true hypertension [7]. It removes the white coat effect. Therefore, using ambulatory blood pressure as an inclusion criterion is able to ensure that renal denervation will not be performed in subjects with white coat hypertension.

Compared with office blood pressure, ambulatory blood pressure provides better estimates of a patient's cardiovascular prognosis. For example, the Systolic Hypertension in Europe (SystEur) trial showed that in the placebo group office blood pressure at baseline was only weakly associated with cardiovascular events and stoke $(\mathrm{P}>0.05)$, whereas the 24-hour ambulatory blood pressure significantly predicted fatal and nonfatal cardiovascular events and stoke [8]. The 24-hour ambulatory blood pressure still predicted the incidence of fatal and nonfatal cardiovascular events and stroke after adjustment for the office blood pressure [8].

In addition, ambulatory blood pressure can provide information on drug non-adherence which is a major limitation in the current clinical trials on renal denervation [9]. Ambulatory blood pressure monitoring provides blood pressure values over a 24-hour period. Therefore, it can provide information on drug non-adherence using the pattern of the blood pressure change after the intake of antihypertensive drugs in renal denervated patients [6]. This advantage of ambulatory blood pressure monitoring has not been explored in clinical trials on renal denervation and it is worthwhile to fully utilize this information in the future. 
In summary, ambulatory blood pressure may be a better inclusion criterion, and changes in ambulatory blood pressure may be designed as the primary efficacy outcome in clinical trials on renal denervation in the future.

\section{Conflict of interest}

The author has no conflicts of interest to disclose.

\section{Acknowledgement}

Y.W. is supported by the National Health and Medical Research Council (1062671). 


\section{References}

[1] Bhatt DL, Kandzari DE, O'Neill WW, et al. A controlled trial of renal denervation for resistant hypertension. N Engl J Med 2014;370:1393-1401.

[2] de la Sierra A, Segura J, Banegas JR, et al. Clinical features of 8295 patients with resistant hypertension classified on the basis of ambulatory blood pressure monitoring. Hypertension 2011;57:898-902.

[3] Fagard RH, Cornelissen VA. Incidence of cardiovascular events in white-coat, masked and sustained hypertension versus true normotension: a meta-analysis. J Hypertens 2007;25:2193-2198.

[4] Mahfoud F, Ukena C, Schmieder RE, et al. Ambulatory blood pressure changes after renal sympathetic denervation in patients with resistant hypertension. Circulation 2013;128:132-140.

[5] Wang Y. Renal denervation for resistant hypertension-the Symplicity HTN-1 study. Lancet 2014;383:1885.

[6] Ruzicka M, McCormick B, Leenen FH, et al. Adherence to blood pressure-lowering drugs and resistant hypertension: Should trial of direct observation therapy be part of preassessment for renal denervation? Can J Cardiol 2013;29:1741.e1741-1743.

[7] Hermida RC, Smolensky MH, Ayala DE, et al. 2013 ambulatory blood pressure monitoring recommendations for the diagnosis of adult hypertension, assessment of cardiovascular and other hypertension-associated risk, and attainment of therapeutic goals. Chronobiol Int 2013;30:355-410.

[8] Staessen JA, Thijs L, Fagard R, et al. Predicting cardiovascular risk using conventional vs ambulatory blood pressure in older patients with systolic hypertension. Systolic Hypertension in Europe Trial Investigators. JAMA 1999;282:539-546. 
[9] Wang Y. Limitations in current clinical trials on renal denervation. Int J Cardiol 2014;174:225. 